\title{
A New Method for Consistency Correction of Judgment Matrix in AHP
}

\author{
Hao Zhang ${ }^{1}$ \\ Hainan Normal University, Haikou 571158, China
}

E-mail:714606560@qq.com

\section{Yingbin $\mathrm{Wei}^{2}$}

Hainan College of Software Technology, Qionghai 571400, China

E-mail:W6337@163.com

\section{Ganhua Yu}

Hainan Normal University, Haikou 571158, China

E-mail:1325715538@qq.com

In order to continue to study the consistency correction of the judgment matrix, we studied the relationship between the inconsistency of the judgment matrix and the perturbation matrix, proposed the concept of the perturbation deviation matrix. Then two new methods for the consistency correction of the judgment matrix are presented based on the convex combination of perturbation deviation matrix. Finally, the new methods were validated by examples and compared with the traditional AHP method.

\section{CENet2017}

\section{2-23 July 2017}

Shanghai, China

\footnotetext{
${ }^{1}$ Speaker

${ }^{2}$ This study is supported by the Nation Natural Science Foundation of China (NSFC) under Grant No.71361008, Science and Technology Agency of Hainan Province under Grant No.DXM2014084 and Key Science and Technology Plan Projects of Hainan Province under Grant No.SQ2014YJJC0012 ${ }^{3}$ Corresponding Author
} 


\section{Introduction}

The Analytic Hierarchy Process (AHP) [1] has been widely applied as a qualitative and quantitative decision-making tool. A successful decision on making process requires the judgment matrix to be consistent. If a judgment matrix fails to fulfill the requirements of consistency, then the weight obtained from the judgment matrix can not be utilized as the basis for making decision. In that case, certain adjustments on the matrix will be further required; therefore, the problem of consistency correction becomes an important research content in the AHP. With the presentation of AHP, there have been rich literature focusing on the consistency correction research. Ma and $\mathrm{Xu}$ [2] proposed a weighted arithmetic mean correction method and two criteria for correction validity. Xu and Wei [3] proposed a weighted geometric mean method. The weighted arithmetic mean and weighted geometric mean method were analyzed and compared in [4]. Xu [5] proposed a weighted arithmetic mean and weighted geometric mean method by analyzing the maximum deviation in the judgment matrix. Some authors proposed the vector correction method and perturbation matrix correction method[6-9]. The consistency of the judgment matrix, the fuzzy judgment matrix and the intuitionistic fuzzy judgment matrix are rectified by the deviation matrix and the vector method [10]. Based on the accelerated genetic algorithm, two kinds of NLP model correction methods were proposed in [11]. Bayesian correction method, Hadamard product induced bias matrix (HPIBM) method and the graph theory correction method were proposed in [12-13]. We have proposed a new algorithm for the consistency test of judgment matrix based on probabilistic statistics and hypothesis testing [14]. Based on the said literature, two new methods for the consistency correction of the judgment matrix based on the convex combination of perturbation deviation matrix are presented hereof to further verify the new methods and make comparison with the traditional AHP.

\section{Preliminaries}

Definition 2.1 [14] Let $A=\left(a_{i j}\right)_{p \times q}$ and $B=\left(b_{i j}\right)_{p \times q}$ be a matrix, if $A^{*} B=\left(c_{i j}\right)_{p \times q}$ is satisfied, the product of $A * B$ is called the Hadamard product of matrix, where $c_{i j}=a_{i j} * b_{i j}$ and $*$ refer to the Hadamard product symbol.

Definition 2.2[14] Let $A=\left(a_{i j}\right)_{n \times n}$ be a judgment matrix and the eigenvector corresponding to the largest eigenvalue $\lambda_{\max }$ is $w=\left[w_{1}, w_{2}, \ldots, w_{n}\right]^{T}$,then the matrix $\bar{A}=\left(w_{i j}\right)_{n \times n}$ is called the characteristic matrix of $A=\left(a_{i j}\right)_{n \times n}$, where $w_{i j}=\frac{w_{i}}{w_{j}}, i, j \in\{1,2, \ldots, n\}$. 
Definition 2.3 [14] Let $A=\left(a_{i j}\right)_{n \times n}$ be a judgment matrix, if $A=\bar{A} * E$,then $E$ is called perturbation matrix of $A$, where $E=\left(\varepsilon_{i j}\right)_{n \times n}$.

Definition 2.4 Let $E=\left(\varepsilon_{i j}\right)_{n \times n}$ be a perturbation matrix of $A$,then

(i) If an element $\varepsilon_{i j}$ in $E$ satisfies $\varepsilon_{i j}-1 \neq 0$, then $\left\{\varepsilon_{i j}\right\}_{n \times n}$ is called perturbation deviation matrix,denoted as $D$;

(ii) If an element $\varepsilon_{i j}$ in $E$ satisfied $\varepsilon_{i j}-1=0$, then $\left\{\varepsilon_{i j}\right\}_{n \times n}$ is called perturbation zero deviation matrix,denoted as $D_{0}$.

Theorem 2.1 If the judgment matrix $A$ satisfies $A=\bar{A} * E$, then $A$ is a completely consistent matrix if and only if $E=D_{0}=\left[\begin{array}{cccc}1 & 1 & \cdots & 1 \\ 1 & 1 & \cdots & 1 \\ \cdots & \cdots & \ddots & \vdots \\ 1 & 1 & \cdots & 1\end{array}\right]$.

Proof. Sufficiency: As $\bar{A}$ is the characteristic matrix of $A, \bar{A}$ satisfies the complete consistency, and the perturbation matrix $E=D_{0}$,according to the Hadamard product definition, we know $A$ satisfies the complete consistency.

Necessity: If $A$ satisfies the complete consistency and $\bar{A}$ also satisfies the complete consistency, according to the Hadamard product definition,so we know $E=D_{0}$.

Definition 2.5 Let $D$ and $D_{0}$ be the perturbation deviation matrix and the perturbation zero deviation matrix respectively,and the convex combination of $D$ and $D_{0}$ can be expressed as $\bar{D}=\lambda D+(1-\lambda) D_{0}$, then $\bar{\varepsilon}_{i j}=\lambda \varepsilon_{i j}+(1-\lambda), \quad \bar{\varepsilon}_{j i}=1 / \bar{\varepsilon}_{i j}$, where, $0 \leq \lambda \leq 1$.

Theorem 2.2 [9] Let inconsistency judgment matrix $A$ be rectified as $A^{\prime}=\bar{A} \circ \bar{D}$,if $\max _{i}\left\{\sum_{j=1}^{n} \varepsilon_{i j}\right\} \leq \lambda_{\max }(D)$,then $\lambda_{\max }\left(A^{\prime}\right)<\lambda_{\max }(A)$.

Definition 2.6 [14] Let $\chi_{1-p}^{2} \sigma_{0}^{2} / n(n-1)$ be a critical value of the consistency index, which is called the Chi- Square Consistency Index(briefly CSCI ). 


\begin{tabular}{ccccccccc}
\hline Order & 3 & 4 & 5 & 6 & 7 & 8 & 9 \\
\hline $\mathrm{p}=0.01$ & 0.010 & 0.036 & 0.064 & 0.087 & 0.106 & 0.121 & 0.134 \\
$\mathrm{p}=0.05$ & 0.029 & 0.068 & 0.099 & 0.121 & 0.138 & 0.151 & 0.162 \\
$\mathrm{p}=0.10$ & 0.049 & 0.092 & 0.122 & 0.138 & 0.158 & 0.169 & 0.178 \\
\hline Order & 10 & 11 & 12 & 13 & 14 & 15 & 16 \\
\hline $\mathrm{p}=0.01$ & 0.144 & 0.153 & 0.160 & 0.166 & 0.172 & 0.177 & 0.181 \\
$\mathrm{p}=0.05$ & 0.170 & 0.177 & 0.183 & 0.188 & 0.192 & 0.196 & 0.199 \\
$\mathrm{p}=0.10$ & 0.185 & 0.191 & 0.196 & 0.200 & 0.204 & 0.207 & 0.210 \\
\hline Order & 17 & 18 & 19 & 20 & 21 & 22 & $/$ \\
\hline $\mathrm{p}=0.01$ & 0.185 & 0.189 & 0.191 & 0.194 & 0.197 & 0.199 & $/$ \\
$\mathrm{p}=0.05$ & 0.202 & 0.205 & 0.207 & 0.209 & 0.211 & 0.213 & $/$ \\
$\mathrm{p}=0.10$ & 0.212 & 0.214 & 0.216 & 0.218 & 0.219 & 0.221 & $/$ \\
\hline
\end{tabular}

Table 1: Critical Value of $C S C I$

If $\left(\lambda_{\max }-n\right) /(n-1)<0.1 R I$, then the matrix through the consistency test;otherwise,not through the consistency test [1]. Where, $C I=\left(\lambda_{\max }-n\right) /(n-1)$, and $0.1 \mathrm{RI}$ is a critical value for $C I$ to test the consistency of the judgment matrix.In this paper,we present $C S C I$ as proposed in [14] as a new critical value of $\left(\lambda_{\max }-n\right) /(n-1)$ to test the consistency of the judgment matrix.

\begin{tabular}{cccccccc}
\hline Order & 3 & 4 & 5 & 6 & 7 & 8 & 9 \\
\hline RI & 0.58 & 0.90 & 1.12 & 1.24 & 1.32 & 1.41 & 1.45 \\
CI=0.1RI & 0.058 & 0.090 & 0.112 & 0.124 & 0.132 & 0.141 & 0.145 \\
\hline Order & 10 & 11 & 12 & 13 & 14 & 15 & $/$ \\
\hline RI & 1.49 & 1.51 & 1.48 & 1.56 & 1.57 & 1.59 & $/$ \\
CI=0.1RI & 0.149 & 0.151 & 0.148 & 0.156 & 0.157 & 0.159 & $/$ \\
\hline
\end{tabular}

Table 2: Critical Value of RI and CI [1]

\section{A new algorithm for consistency correction}

It is proposed to replace $d_{i j}$ in the perturbation matrix with $\gamma d_{i j}+(1-\gamma)$, and the Hadamard product $\left(w_{i} / w_{j}\right) *\left[\gamma d_{i j}+(1-\gamma)\right]$ is used as the correction result of the element in the original matrix[9].However, considering there are too many elements for each correction in [9],it is difficult to maintain the information in the original matrix. In this paper, we propose a consistency correction method, which is easy to maintain the information in the original matrix.

Let $A=\left(a_{i j}\right)$ be an $n \times n$ judgment matrix, $k$ be the number of iterative times, and the specific steps are as follow:

Step 1: Let $A^{(0)}=\left(a_{i j}^{0}\right), \theta \in(0,1)$, and $k=0$. 
Step 2: Calculate the maximum eigenvalue $\lambda_{\max }^{(k)}$ of $A^{(k)}$ and the priority vector $w^{(k)}=\left(w_{1}^{(k)}, w_{2}^{(k)}, \ldots, w_{n}^{(k)}\right)^{T}$.

Step 3: If $\left(\lambda_{\max }^{(k)}-n\right) /(n-1)<C S C I$,then go to Step 6; otherwise, go to next step.

Step 4:

(i) (Method 1)

Normalize all columns of $A^{(k)}$,then get the normalized matrix $\bar{A}^{(k)}=\left(a_{1}^{(k)}, a_{2}^{(k)}, \cdots, a_{n}^{(k)}\right)$, in which, refers to the column vector of $\bar{A}^{(k)}$. Calculate the cosine value of the included angle between $w^{(k)}$ and $a_{i}^{(k)}$, namely $\cos \theta_{i}^{(k)}=\frac{\left(w^{(k)}, a_{i}^{(k)}\right)}{\left|w^{(k)}\right|\left|a_{i}^{(k)}\right|}$.

Then determine $t$ so that $\cos \theta_{t}^{(k)}=\min _{i}\left\{\cos \theta_{i}^{(k)}\right\}$, and let $A^{(k+1)}=\left(w_{i}^{(k)} / w_{j}^{(k)}\right) * \varepsilon_{i j}^{(k)}$, where

$$
\varepsilon_{i j}^{(k)}=\left\{\begin{array}{c}
\theta \varepsilon_{i t}^{(k)}+(1-\theta), j=t \\
\frac{1}{\theta \varepsilon_{t j}^{(k)}+(1-\theta)}, i=t \\
\varepsilon_{i j}^{(k)}, \quad i, j \neq t
\end{array}\right.
$$

(ii) (Method 2)

Let $\varepsilon_{i j}^{(k)}=a_{i j}^{(k)}\left(w_{j}^{(k)} / w_{i}^{(k)}\right)$ and determine $p, q$ so that $\varepsilon_{p q}^{(k)}=\max _{i, j}\left\{\varepsilon_{i j}^{(k)}\right\}$. Let $A^{(k+1)}=\left(w_{i}^{(k)} / w_{j}^{(k)}\right) * \varepsilon_{i j}^{(k)}$, where

$$
\varepsilon_{i j}^{(k)}=\left\{\begin{array}{cc}
\theta \varepsilon_{p q}^{(k)}+(1-\theta), & (i, j)=(p, q) \\
\frac{1}{\theta \varepsilon_{p q}^{(k)}+(1-\theta)}, & (i, j)=(q, p) \\
\varepsilon_{i j}^{(k)}, & (i, j) \neq(p, q),(q, p)
\end{array}\right.
$$

Step 5: Let $k=k+1$, and return to Step 2 .

Step 6: Output $A^{(k)}, \lambda_{\max }, C I, w^{(k)}$, in which, refers to the correction matrix and refers to the vector of priorities.

Step 7: End.

The two criteria for measuring the proximity of the original matrix to the correction matrix are given in [2],as follows:

$$
\begin{aligned}
\delta^{(k)} & =\max _{i, j}\left\{\left|a_{i j}^{(k)}-a_{i j}^{(0)}\right|\right\}, i, j \in N \\
\sigma^{(k)} & =\sqrt{\sum_{i=1}^{n} \sum_{j=1}^{n}\left(a_{i j}^{(k)}-a_{i j}^{(0)}\right)^{2}} / n
\end{aligned}
$$


Usually we think that the smaller the value of $\delta^{(k)}, \sigma^{(k)}$ is, the more information will be retained from the original matrix and the better correction will be achieved.

\section{Case analysis}

This paper chooses the Matrix $A$ in [2] and rectifies it according to the above two methods, as follows:

$$
A=\left[\begin{array}{cccccccc}
1 & 5 & 3 & 7 & 6 & 6 & 1 / 3 & 1 / 4 \\
1 / 5 & 1 & 1 / 3 & 5 & 3 & 3 & 1 / 5 & 1 / 7 \\
1 / 3 & 3 & 1 & 6 & 3 & 4 & 6 & 1 / 5 \\
1 / 7 & 1 / 5 & 1 / 6 & 1 & 1 / 3 & 1 / 4 & 1 / 7 & 1 / 8 \\
1 / 6 & 1 / 3 & 1 / 3 & 3 & 1 & 1 / 2 & 1 / 5 & 1 / 6 \\
1 / 6 & 1 / 3 & 1 / 4 & 4 & 2 & 1 & 1 / 5 & 1 / 6 \\
3 & 5 & 1 / 6 & 7 & 5 & 5 & 1 & 1 / 2 \\
4 & 7 & 5 & 8 & 6 & 6 & 2 & 1
\end{array}\right] .
$$

The perturbation matrix of the original judgment matrix is obtained as follows:

$$
\begin{gathered}
{\left[\begin{array}{cccccccc}
1 & 1.5599 & 3.2612 & 0.7076 & 1.0766 & 1.2591 & 0.3213 & 0.4813 \\
0.6411 & 1 & 1.1614 & 1.6200 & 1.7252 & 2.0178 & 0.6180 & 0.8816 \\
0.3066 & 0.8610 & 1 & 0.5579 & 0.4951 & 0.7722 & 5.3280 & 0.3542 \\
1.4132 & 0.6173 & 1.7923 & 1 & 0.5916 & 0.5190 & 1.3624 & 2.3809 \\
0.9290 & 0.5796 & 2.0197 & 1.6903 & 1 & 0.5848 & 1.0746 & 1.7886 \\
0.7942 & 0.4956 & 1.2951 & 1.9269 & 1.7100 & 1 & 0.9188 & 1.5292 \\
3.1120 & 1.6182 & 0.1879 & 0.7340 & 0.9306 & 1.0884 & 1 & 0.9986 \\
2.0775 & 1.1343 & 2.8230 & 0.4200 & 0.5591 & 0.6539 & 1.0014 & 1
\end{array}\right], \text {, }} \\
\lambda_{\max } \approx 9.6689, \frac{\lambda_{\max }-n}{n-1}=0.2384>C S C I, \mathrm{w}=(0.1730,0.0540,0.1881,0.175,0.0310,0.0363,
\end{gathered}
$$

$0.1668,0.3332)$.

When $\mathrm{p}=0.10, \theta=0.5$, the correction result in Method 1 is:

$A^{(k)}=\left[\begin{array}{cccccccc}1 & 4.9998 & 2.0811 & 6.9998 & 6.0000 & 6.0000 & 0.6853 & 0.2500 \\ 0.2000 & 1 & 0.3524 & 5.0000 & 3.0000 & 3.0000 & 0.2618 & 0.1429 \\ 0.3333 & 3.0000 & 1 & 5.9995 & 2.9997 & 4.0004 & 3.5638 & 0.2000 \\ 0.1429 & 0.2000 & 0.1404 & 1 & 0.3333 & 0.2500 & 0.1239 & 0.1250 \\ 0.1667 & 0.3333 & 0.2688 & 3.0001 & 1 & 0.5000 & 0.1930 & 0.1667 \\ 0.1667 & 0.3333 & 0.2472 & 4.0000 & 2.0000 & 1 & 0.2088 & 0.1667 \\ 3.0000 & 5.0000 & 0.6617 & 7.0003 & 5.0004 & 4.9999 & 1 & 0.5000 \\ 3.9999 & 6.9998 & 3.5899 & 8.0005 & 5.9997 & 6.0000 & 1.9986 & 1\end{array}\right]$,

$$
\lambda_{\max } \approx 8.7633, \frac{\lambda_{\max }-n}{n-1}=0.1090<0.121=C S C I, w^{(k)}=(0.1832,0.0651,0.1289,0.0182,
$$

$0.0326,0.0412,0.1954,0.3354), \delta^{(k)}=2.4362, \sigma^{(k)}=0.2240$.

When $\mathrm{p}=0.10, \theta=0.5$, the correction result in Method 2 is: 


$$
A^{(k)}=\left[\begin{array}{cccccccc}
1 & 5.0000 & 3.0000 & 7.0000 & 6.0000 & 6.0000 & 0.3333 & 0.2500 \\
0.2000 & 1 & 0.3333 & 5.0000 & 3.0000 & 3.0000 & 0.2000 & 0.1429 \\
0.3333 & 3.0000 & 1 & 6.0000 & 3.0000 & 4.0000 & 1.5486 & 0.2000 \\
0.1429 & 0.2000 & 0.1667 & 1 & 0.3333 & 0.2500 & 0.1429 & 0.1250 \\
0.1667 & 0.3333 & 0.3333 & 3.0000 & 1 & 0.5000 & 0.2000 & 0.1667 \\
0.1667 & 0.3333 & 0.2500 & 4.0000 & 2.0000 & 1 & 0.2000 & 0.1667 \\
2.0474 & 5.0000 & 0.1667 & 7.0000 & 5.0000 & 5.0000 & 1 & 0.5000 \\
4.0000 & 7.0000 & 5.0000 & 8.0000 & 6.0000 & 6.0000 & 2.0000 & 1
\end{array}\right],
$$

$$
\lambda_{\max } \approx 8.8077 \quad, \quad \frac{\lambda_{\max }-n}{n-1}=0.1154 \quad<\quad 0.121=\text { CSCI }
$$

$w^{(k)}$

0.0342,0.0415,0.1793,0.3543), $\delta^{(k)}=3.4514, \sigma^{(k)}=0.4476$.

If $0.1 \mathrm{RI}$ in the traditional AHP is used as the critical value of $\frac{\lambda_{\max }-n}{n-1}$, then the correction result in Method 1 is:

$$
A^{(k)}=\left[\begin{array}{cccccccc}
1 & 5.0000 & 3.0001 & 7.0005 & 5.9998 & 5.9994 & 0.9136 & 0.2500 \\
0.2000 & 1 & 0.3333 & 5.0001 & 2.9998 & 3.0002 & 0.3061 & 0.1429 \\
0.3333 & 3.0001 & 1 & 5.9994 & 2.9997 & 4.0000 & 1.4626 & 0.2000 \\
0.1429 & 0.2000 & 0.1667 & 1 & 0.3333 & 0.2500 & 0.1030 & 0.1250 \\
0.1667 & 0.3333 & 0.3334 & 3.0001 & 1 & 0.5000 & 0.1776 & 0.1667 \\
0.1667 & 0.3334 & 0.2500 & 4.0000 & 2.0000 & 1 & 0.2085 & 0.1667 \\
3.0001 & 5.0000 & 0.1667 & 7.0001 & 5.0007 & 4.9999 & 1 & 0.5000 \\
3.9999 & 7.0000 & 5.0000 & 7.9997 & 6.0003 & 5.9997 & 1.8752 & 1
\end{array}\right],
$$

$\lambda_{\max }=8.9640, \frac{\lambda_{\max }-n}{n-1}=0.1377<0.141=0.1 \mathrm{RI}, w^{(k)}=(0.1900,0.0626,0.1227,0.0182,0.032$ 8, 0.0294,0.1971,0.3472), $\delta^{(k)}=4.5374, \sigma^{(k)}=2.6497$.

If $0.1 \mathrm{RI}$ in the traditional AHP is used as the critical value of $\frac{\lambda_{\max }-n}{n-1}$, then the correction result in Method 2 is:

$$
\begin{gathered}
A^{(k)}=\left[\begin{array}{cccccccc}
1 & 4.9999 & 3.0001 & 7.0004 & 5.9999 & 6.0000 & 0.3334 & 0.2500 \\
0.2000 & 1 & 0.3333 & 5.0002 & 3.0001 & 3.0000 & 0.2000 & 0.1429 \\
0.3333 & 3.0001 & 1 & 5.9994 & 2.9997 & 4.0000 & 1.4755 & 0.1988 \\
0.1429 & 0.2000 & 0.1667 & 1 & 0.3333 & 0.2500 & 0.1429 & 0.1250 \\
0.1667 & 0.3333 & 0.3334 & 3.0000 & 1 & 0.5000 & 0.2000 & 0.1667 \\
0.1667 & 0.3333 & 0.2500 & 4.0000 & 2.0000 & 1 & 0.2000 & 0.1667 \\
3.0000 & 4.9999 & 0.1667 & 6.9995 & 5.0001 & 5.0000 & 1 & 0.5000 \\
3.9999 & 7.0001 & 5.0000 & 8.0003 & 6.0006 & 5.9990 & 2.0001 & 1
\end{array}\right], \\
\lambda_{\max } \approx 8.9058, \frac{\lambda_{\max }-n}{n-1}=0.1294<0.141=0.1 \mathrm{RI}, w^{(k)}=(0.1784,0.0613,0.1239,0.0191, \\
0.0337,0.0407,0.1950,0.3430), \delta^{(k)}=4.5245, \sigma^{(k)}=0.5656 .
\end{gathered}
$$


By contrast, it is found that the value of $\delta^{(k)}$ and $\sigma^{(k)}$ in the new correction method is smaller than the corresponding traditional correction method, so the new correction method is better.

\section{Conclusion}

In this paper, we studied the relationship between the perturbation matrix and the judgment matrix inconsistency, proposed two new methods of consistency correction based on the theory of perturbation deviation matrix. The element to be rectified are determined by the size of the disturbance element in the perturbation matrix and the size of the cosine between the vectors. Two new methods have been verified by the satisfied results.

\section{References}

[1] Saaty T L. The analytic hierarchy process[M].New York: McGraw-Hill,1980.

[2] Ma Weiye,Xu Jiangyue,Wei Yixiang.A practical approach to modifying pairwise comparison matrices and two criteria of modificatory effectiveness[J].Journal of systems science \& systems ngineering,1993,2(4):334-338.

[3]Xu Zeshui,Wei Cuiping.A consistency improving method in the analytic hierarchy process[J]. European journal of operational research,1999,116:443-449.

[4] Xu Zeshui,Da Qingli.Analysis and comparison of two methods for improving consistency of judgment matrix[J]. Journal of Southeast university(Natural Science Edition), 2002,32(6):913 -916 .

[5] Xu Zeshui. A Practical method for improving consistency of judgment matrix in the AHP[J]. Journal of systems science and complexity,2004,17(2):169-175.

[6] Liu Wanli,Lei Zhijun.Study on Rectification method for the judgment matrix in $A H P[J] . S y s t e m s \quad$ Engineering-Theory\&Practice,1997,(6): 30-34.(In Chinese)

[7] Xu Zeshui.Two approaches to improving the consistency of complementary judgement matrix [J]. $\quad$ App. Math.J.Chinese Univ.Ser.B,2002,17(2):227-235.

[8] Zhang Qunhui,Long Xihua.A iterative algorithm for improving the consistency of judgment matrix in AHP[J]. Mathematics in practice and theory,2001,31(5): 565-568.(In Chinese)

[9] Dong Cao,Lawrence C.Leung,Japhet S.Law. Modifying inconsistent comparison matrix in analytic hierarchy process:A heuristic approach[J].Decision support systems,2008,44:944-953.

[10] Weixia Li,Chengyi Zhang.Decision-Making Interactive and Iterative Approaches[M].

Switzerland: Springer international publishing,2015,219-244.

[11] Yucheng Dong,Yihua Chen,Jian Xiao.Two new method for improving the consistency of the judgement matrix in AHP[J].Journal of systems science and information,2005,3(3):501-508.

[12] Changsheng Lin,Gang Kou.Bayesian revision of the individual pairwise comparison matrices under consensus in AHP-GDM[J].Applied soft computing,2015,35:802-811. 
[13] Gang Kou,Daji Ergu,Jennifer Shang.Enhancing data consistency in decision matrix:Adapting Hadamard model to mitigate judgement contradiction[J].European journal of operational research,2014,236:261-271.

[14] Ganhua Yu ,Yajie Zhang,Haijing Huang,Chengyi Zhang.A New Method for Consistency Test of Judgment Matrix in AHP[J].Mathematics in practice and theory,(Accepted).(In Chinese) 